\title{
CARE - Extending a Digital Picture Frame with a Recommender Mode to Enhance Well-Being of Elderly People
}

\author{
Thomas Rist*, Andreas Seiderer ${ }^{\dagger}$, Stephan Hammer ${ }^{\dagger}$, Marcus Mayr* and Elisabeth André ${ }^{\dagger}$ \\ *University of Applied Sciences Augsburg, Germany \\ ${ }^{\dagger}$ Augsburg University, Germany
}

\begin{abstract}
This contribution addresses the development of technology for the elderly with the aim to improve their general well-being. We report work on a prototype system named CARE that combines the functionality of a digital image frame with an active recommender mode. The purpose of the recommender is to provide the user with context-specific recommendations. Recommendations are chosen on the basis of data acquired by sensors embedded in the user's environment, and a well-being model to carefully decide on at which point in time what kind of activity is most suitable to suggest. We describe conception and implementation of a first CARE prototype that we used for in-situ testing in a senior's home, as well as for gathering feedback on system usage and user appreciation. The paper concludes with some lessons learned from analyzing log file data of the trial period and from conducting a post-trial interview.
\end{abstract}

Keywords-Well-being and lifestyle support, pervasive healthcare for the elderly, usability and acceptability

\section{INTRODUCTION}

Increases in longevity and improvements in health care services together with declining birth rates contribute to the irresistible phenomenon of an aging society in almost all western countries. While higher life expectancy is a positive achievement, it comes along with societal challenges to be dealt with urgently, among those are:

- Social isolation and lack of societal participation of solitarily living elderly people

- $\quad$ Loss of living independence due physical setbacks and limited mobility

- Inactivity and missing initiative of elderly people

Lifestyle choices are among the most important factors that determine a person's well-being. Studies have shown that regular physical exercise can help mitigate many age-related diseases [1]. Furthermore, there is evidence that creative activities, such as gardening or painting, have a positive influence on people's well-being [2]. Previous studies conducted in a senior citizen's apartment building have shown that interventions that encourage the elderly to participate in activities, such as taking over responsibilities for daily household chores, are effective means to prevent social isolation and loneliness [3]. A meta study on interventions that contribute to well-being is provided by Catan et al. [4].

The objective of CARE (Context-Aware Recommender system for the Elderly) is to develop a sensitive and personalized assistance system to support single-living people in their domestic environment. Assistance is given in the form of situationally appropriate and unobtrusive recommendations to encourage physical, mental and social activities. To this end, CARE combines the concept of a classical digital picture frame with a recommender system. That is it interleaves the display of photos with situation-specific recommendations, all aimed at increasing individual quality of life and vitality. The recommender system is triggered by user presence while the picture frame mode is active when the user is further away from the display.

The current paper reports work on a first CARE prototype system. After discussing related work, we provide an overview of the overall CARE concept and its implementation in terms of soft- and hardware. The main purpose of prototype implementation was to have a concrete device for in-situ testing in a senior's home and feedback gathering on system usage and user appreciation. A first trial run of CARE has been conducted over a period of two weeks. The paper concludes with some lessons learned from analyzing log file data and from conducting a post-trial interview.

\section{RELATED WORK}

There are a growing number of projects that aim to promote a healthier lifestyle and to increase quality of life and overall well-being. Many systems rely on self-monitoring as a means to evoke a behavior change in people. Typically, health-related data are presented to the user in terms of diagrams, such as line or bar charts, to make the users reflect on them. Thereby, data are either manually collected by the users themselves or automatically acquired from body-worn sensors, sensors embedded in the environment or in the users' mobile phone.

Since many users have difficulties to draw relevant conclusions from such diagrams, attempts have been made to convey health-related data in a form that is easier to grasp. The BeWell system [5] presents different well-being dimensions, such as the users' physical activities or their sleeping patterns, by different characters in an aquatic ecosystem while the UbiFit system [6] employs the metaphor of a virtual garden that reflects the user's physical fitness. To show the users detected connections between their lifestyle and their well-being, the Health Mashups system [7] makes use of natural language. For example, based on information acquired from mobile sensor data, users may be told that they tend to feel better if they have more sleep. The information provided to the user is derived from personal user and context data collected with sensors 
integrated into the users' mobile phone. The Motivate system [8] maps health-related data about user and context directly onto situated recommendations based on the consideration that advice is more likely to be followed if it is presented at the right location and the right point in time. Furthermore, studies have been conducted with virtual [9]-[11] or robotic agents [9] that serve as lifestyle or health advisors. There is evidence that elderly people respond positively to such companions in particular if they emulate social behaviors. However, as Vardoulakis et al. [12] pointed out elderly people also expect a large variety of joint social activities, such as small talk, playing cards etc., from the robot resulting into a rather timeconsuming and technically challenging implementation.

Besides different modalities, such as diagrams or natural language, a variety of stationary and mobiles devices has been explored to convey health and lifestyle related information to users. While mobile devices might be very useful to seniors, they also raise a number of usability issues because potential physical and cognitive impairments of elderly people are not adequately addressed [13]. According to Leung et al. [14], many adults aged 65+ have difficulties learning how to use mobile phones. An evaluation by Jones et al. [15] of older people's use of iPads in care homes revealed that iPads may increase the engagement and motivation of older people. However, they also mentioned the weight of iPads as a potential barrier of use. Motti et al. [16] pointed out that touch-based interaction may alleviate barriers of getting started for elderly people, but at the same time they detected a number of usability issues, such as the timing of tapping gestures. Leonardi et al. [17] surveyed a large number of publications on smarthome technology for promoting health and well-being of older adults. As a major barrier of acceptance, they identified privacy and security issues.

The rationale behind so-called ambient displays is to present users with information on displays that are smoothly integrated into their daily environment. Mateevitsi et al. [18] made use of a health bar installed below the monitor of office workers to make them aware of their sitting habits and encourage them after prolonged sitting periods to interrupt their work for a short walk. Another approach to improve the sitting habits of office workers has been presented by Obermair et al. [19] who set up picture frames in an office environment. The picture frames included animated portraits of known or beloved people who provided affective feedback on the employees' postures.

The concept of a digital picture frame has also been researched to enhance awareness within a family of a senior's daily life by augmenting a portrait of the elderly person with data related to health and well-being, see the work by Rowan and Mynatt [20] or Consolvo et al. [21]. Lindley et al. [22] made use of a display with different channels each of them showing a photo of a family member to give elderly people a possibility to initiate contact with them. Cornejo et al. [23] combined a lightweight face book client with social exer games on an interactive display to encourage social encounters of seniors with family members. López-Nores et al. [24] explored options to incorporate health-related recommendations into semantically matching TV programs.

Overall, assistive technologies for the elderly mainly employed the concept of a digital picture frame to improve the seniors' safety and to encourage interactions within their social network. Our work makes use of a digital picture frame as well, but combines it with an ambient display for the personalized presentation of recommendations related to health and wellbeing. Our research specifically focuses on seniors from the $65+$ age group as target users. This has various implications with regards to sensor data acquisition, data interpretation, and the kind of recommendations that CARE delivers to its users.

\section{OVERVIEW OF THE CARE SYSTEM}

CARE capitalizes on the concept of a digital picture frame as such devices are often given to senior family members. However, CARE makes a substantial extension to the classical concept. It interleaves the display of photos with physical exercises, brain-twisters and situation-specific life style recommendations, in other words, it combines two modes:

- Picture frame mode: This mode is activated when the user is further away from the display. In this mode, CARE appears to the user like an ordinary picture frame which displays photos that are either taken from a local repository, or which are remotely uploaded by family members via an internet connection. Users implicitly get information through a quick glance. However, they neither control the display of pictures nor do they interact with the system.

- Recommender mode: This mode is triggered by user presence detected in front of the display. In this mode, users receive context-specific recommendations. Recommendations are chosen on the basis of data acquired by sensors embedded in the user's environment and possibly body-worn sensors as well as a user model, a discourse model, and a model of well-being factors to carefully decide on at which point in time what kind of activity will be most suitable to suggest in order to increase the user's well-being.

In order not to overstrain users, interaction with the display is deliberately kept simple. Users are able to interact with the display, for example, by selecting buttons to answer quizzes or by physically interacting with the magnets of the board in which the tablet has been integrated (see Section III-C). Furthermore, they can provide feedback on presented recommendations and indicate how they feel by selecting specific icons on the screen.

\section{A. Modeling Well-Being}

A number of attempts have been made to define what factors constitute a person's subjective feeling of well-being, and to identify suitable indicators for measuring subjective well-being, e.g. for the purpose of comparisons. As it is often the case with high-level socio-physiological constructs, there is little consensus regarding the definition of this construct and its differentiation from related constructs, such as "Happiness", or "Quality of Life", which is in focus of various studies in the medical domain. The great diversity in the definition of constructs and models is mirrored by the broad range of different survey questionnaires and online tools for measuring a person's degree of well-being, happiness, or health-relatedquality of life. A collection of surveys for self testing can, for example, be found on the website of the TES organization (https://www.energystrategy.eu/free-well-being-surveys). 
A profound comparison of models is beyond the scope of our work. Rather, we take a pragmatic approach and rely on existing frameworks as an inspiration for our work towards a CARE prototype. One of these frameworks is a model that has been put forward by the new economic foundation (nef). On their web site (www.nationalaccountsofwellbeing.org) they report on an activity which aimed to measure subjective wellbeing of citizens living in different European countries. Their underlying conceptualization of well-being is based on personal well-being and social well-being as headline indicators, each of which is broken further down into a number of suband sub-sub-categories. At the lowest level of this hierarchy, categories are linked to concrete items of a questionnaire that can serve as a measuring tool in well-being studies. Another framework is the SCL/PRB Index of Well-Being in Older Populations developed by the Stanford Center on Longevity (SCL) and the Population Reference Bureau (PRB) [25] which characterizes Well-Being by four domains (Material WellBeing, Physical and Cognitive Well-Being, Social Engagement and Emotional Well-Being) and indicators that are used to provide a score for each domain.

For the purpose of CARE, we rely on a hierarchical model of well-being factors as the nef framework. For practical reasons, however, we use a simplified model. It includes only categories which are linked to recommendations that a system like CARE is able to deal with. For instance, CARE can make suggestions about how to improve physical fitness, but it would be difficult to provide recommendations regarding a senior's income which is considered a dominating factor for material well-being. Our current model comprises the following headline categories: physical, mental, social, emotional, and environmental well-being. The headline categories are inspired by the domains of the SCL/PRB Index of WellBeing in Older Populations. However, we use the term mental well-being instead of cognitive well-being. Furthermore, we include environmental well-being as an additional category which refers to all environmental factors that may affect human well-being, such as air quality or room climate.

As shown in Fig. 1, these categories are further broken down into subcategories. In the context of CARE the purpose of a well-being model is to associate with its subcategories a set of dedicated recommendations that may be selected for presentation to a senior, in the hope that recommendations are followed and that recommended activities eventually contribute positively to well-being, (see Section III-B).

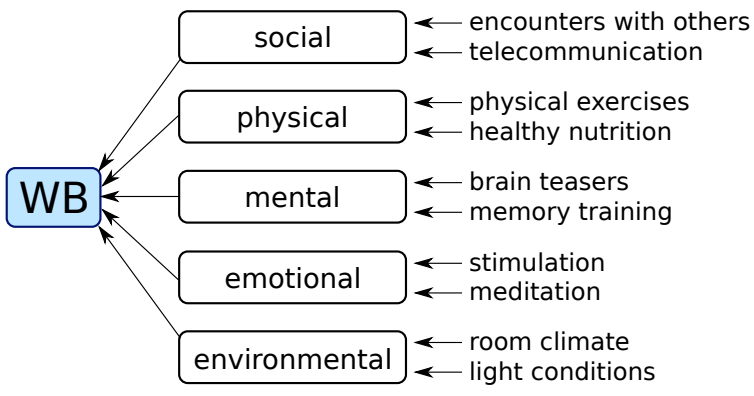

Fig. 1. CARE's model of factors contributing to well-being

To check plausibility of CARE's well-being model we conducted structured interviews among a peer-group of 21 seniors (13 female, 8 male, in the age range between 67 and 83 ), all living either alone or together with a partner in an own household. The structured interviews started with a number of demographic questions (including age, gender, education, marital status, accommodation, and living arrangements) and questions on usage of electronic media (TV, radio, computer, internet, and digital picture frames) at home. An important outcome for the development of CARE was that all participating seniors had an idea about what a digital picture frame is, though only two of them had one at home.

The interviews continued with specific questions related to the subcategories of CARE's well-being model. The objective of this part was to learn about:

- the activities that seniors already perform regularly, and that could relate to the model's subcategories

- what importance the seniors attach to those activities

- whether the seniors have set specific targets to engage more strongly / often in those activities

- whether the seniors see specific obstacles that may hinder stronger engagement

For illustration, let's focus on the subcategory physical well-being. This subcategory depends, among other factors, on a person's physical fitness (or physical vitality), the general health condition, and feeling of vigilance.

Physical fitness may be measured by indicators, such as endurance, strength, coordination, and suppleness. To assess a person's physical fitness, we asked subjects which kind of physical activity they perform and how frequently they do so. We also asked whether it is important to them to be physically active, whether they strive for enhancing their physical fitness to improve their overall well-being, and whether they face specific obstacles (e.g. indispositions) which hinder them to engage more in physical activities. Comparing responses to questions on performed activities with a person's selfassessment of physical fitness on a 6-point scale $(1=$ not fit at all, $6=$ very fit) confirmed that those who engage frequently in physical activity (e.g. riding a bicycle, climbing stairs, go out for walks) feel physically more fit than others.

To assess people's feeling of vigilance we asked the seniors how often they feel tired during the day, and how frequently they suffer from insomnia. We also asked whether they take any counter measures (such as drinking coffee, going out to breath fresh air, taking a shower, or having a nap). Also, most seniors said that they wished to improve their physical wellbeing by means of engaging more in physical activities and by paying more attention to healthy nutrition. As hindering obstacles the seniors mentioned physical impairments at first place, but also lack of time due to other obligations (e.g. caring for relatives or their pets). These results are in line with a study performed by Fan et al. [26]. Interestingly, the survey also revealed that "healthy food" matters when speaking about well-being. Therefore, our model considers "healthy nutrition" as a contribution factor to physical well-being.

For mental well-being we assume mental fitness as a determining factor with measurable indicators, such as capacity for remembering, creativity, logical thinking and inference capability. We did not ask subjects for a self-assessment of 
mental fitness but asked them how frequently they forget names, phone numbers, and dates, or need to search for objects because they forgot where they placed them. Most seniors said that they engage in specific activities to improve mental fitness (such as solving quizzes or crossword puzzles, or memorizing numbers). Factors negatively affecting memory and concentration mentioned by the seniors included headaches, uneasy sleep, stress, medicaments and age.

With regard to social well-being, we asked questions concerning the frequency of face-to-face encounters with relatives, friends, and neighbors, how often they receive and make phone calls, and how often they go out for attending public events. Here we also asked how often they feel being alone, and whether they wished to intensify their social contacts. As barriers to meeting people, the seniors mentioned missing means of transport, tiredness and weariness.

Regarding the subcategory environmental well-being we asked how satisfied they were with their housing condition and their living environment (e.g., the opportunities for shopping, medical treatment, recreation and leisure activities, and contentedness to public transportation).

For the subcategory emotional well-being we only included a few general questions, such as whether they perform relaxation or meditation exercises.

In principle, responses to the indicators of certain wellbeing categories could be aggregated into numeric scores, and at the highest level of the hierarchy, into a single number representing an overall score for a person's well-being. Such scores could be used for measuring the success of a system like CARE. However, this was beyond the objective of our current work.

\section{B. Recommendations to improve well-being}

Our fundamental working hypothesis is that recommendations can positively contribute to a senior's well-being provided that:

- they are of relevance for the person at the time when they are given,

- the person is able to follow them,

- the person is motivated to do so.

Starting from the well-being model introduced above we prepared a repository of about 50 recommendations and linked them to the subcategories of our well-being model. Each recommendation is a ready-for-display media asset, such as a textpicture combination, or a text with an embedded video clip. To give an impression, Fig. 2 shows two recommendations.

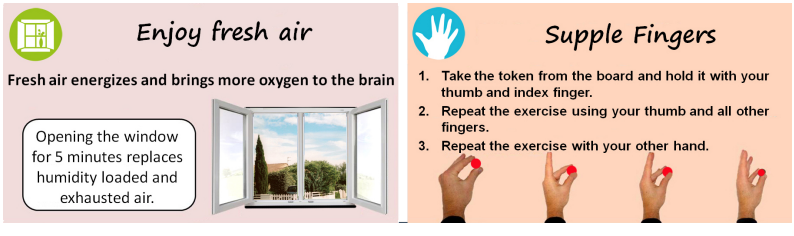

Fig. 2. Two sample recommendations as shown by CARE

To serve their purpose, the elements of a recommendation are associated with certain communicative functions.
For example, the recommendation shown in the right-hand part of Fig. 2 instructs a finger exercise. The instructions about what to do are given by the text. The images of a hand provide additional information on how to perform the exercise. In order to facilitate the recognition of the type of a recommendation, we use a common headline text, a logo and a certain background color for recommendations of a certain type, such as finger exercises. The recommendation shown in the left-hand part of Fig. 2 may be triggered in case of indoor air quality falls below a certain threshold. It is a recommendation to air the room as suggested by the image of an open window. There is no textual request to air the room. Rather, the headline and the text just provide motivating arguments for letting in fresh air.

Recommendations linked to other subcategories of CARE's well-being model include suggestions for healthy nutrition, quizzes and memorization exercises and relaxation. In [27], more information can be found on the repertoire of recommendations and the sources from where they have been compiled.

\section{Set-up of the 1st Care Prototype}

Fig. 3 gives an impression of the CARE prototype. Its hardware consists of a tablet computer with a touch screen (a low-priced HP Omni 10 5600eg running Windows 8.1) that has been integrated into a magnetic pin board. For the purpose of testing, the board is mounted on two rails so that the height of the display screen can be adjusted for its users. The tablet itself has the following integrated sensors: accelerometer, gyroscope, microphone, light sensor, compass, camera and inclinometer. In addition, the pin board is equipped with further sensors. An infrared (IR) distance sensor is used to detect the presence of a person in front of the device within a detection range of 20 to $150 \mathrm{~cm}$. A magnetic field sensor and a sensor for measuring air quality are mounted at the backside of the device. The magnetic field sensor (a 3-axis magnetometer) detects changes in the magnetic field that occur when magnets are removed from or added to the pin board. For measuring indoor air quality we rely on an iAQ-engine sensor which is embedded in a USB stick and connected to CARE.

The right-hand part of Fig. 3 shows the core software components and knowledge sources of the CARE system. The sensor acquisition component interfaces with all attached sensors and collects multi-sensory data with sensor-specific sampling rates. To reduce the volume of raw data, sensor readings with sampling rates higher than $2 \mathrm{~Hz}$ are aggregated (using aggregation functions, such as max, min, mean, median, or sum). Aggregation is also applied for privacy reasons. For example, from recorded audio data CARE only considers intensity-related audio features, such as averaged acoustic amplitudes, for further processing.

We use dedicated classifiers and mechanisms for fusing sensor data to obtain relevant context information. For example, CARE must be aware of whether a person is in the room where the system is installed and whether a person is getting close to the CARE installation. To this end, the following sensor readings are exploited: IR distance, noise level, air quality, temperature, humidity, brightness, magnetic field and the tablet's acceleration, angular velocity, magnetic heading and tilt. The presence of a person in the room may 
be detected by a higher level of background noise, and after a certain time also by a higher concentration of carbon dioxide and volatile organic compounds which are recorded by the air quality sensor. In the evening hours and during the night, an abrupt change of illumination provides further evidence of someone entering or leaving the room. From the readings of the IR distance and the light sensor it can be inferred that a person is in the vicinity of the CARE installation, and thus may take notice of what is displayed on the screen. Also, physical interaction with the magnetic pin board can be detected by the magnetometer, compass, accelerometer, inclinometer, and gyroscope. Although CARE is equipped with a camera, we do not rely on image processing for activity recognition. Rather, if at all, camera images of users are only taken for evaluation purposes and in consensus with the user, see Section IV-B.

A central task of CARE is to decide on when to switch from picture frame mode to recommender mode. For the sake of simplicity, the first CARE prototype switches to recommender mode every time a user is detected in front of the display for a minimum of two seconds.

When switching to recommender mode, the recommender module is called to select a recommendation for display. The selection approach is a two-step process. First a rule-based contextual pre-filtering approach [28] is applied to filter out inadequate recommendations. It considers the current time of day (morning, forenoon, lunchtime, afternoon, evening, night), the local sunrise and sunset times and the current weather conditions (very_Good, Good, Fair, Bad, very_Bad). For example, if it is dark outside, all outdoor activities, such as "take a walk", are filtered out. After the pre-filtering process, the recommender system chooses a single recommendation while taking into account the display history. Furthermore, the recommender module is able to immediately react on particular situations by triggering a corresponding rule. For example, if the trigger event refers to bad air quality in the room, the module selects a recommendation of category "environmental well-being". In case of "bad_user_mood" is detected, recommendations of category "emotional well-being" have increased relevance and will get selected with high probability.
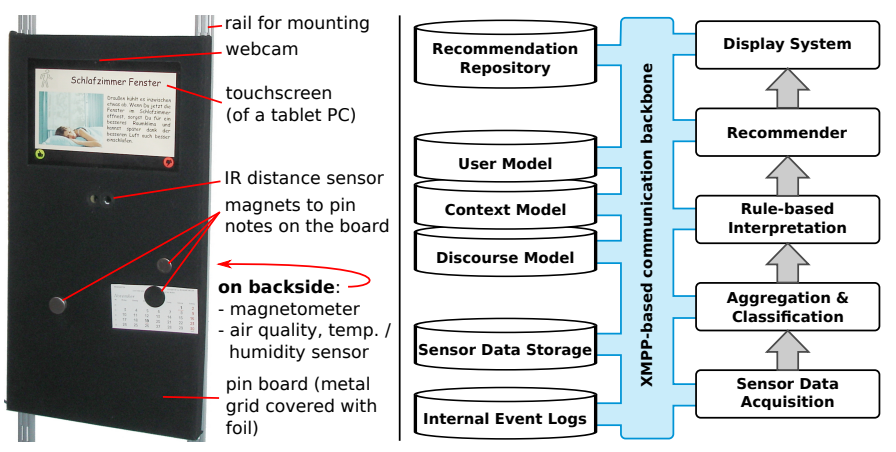

Fig. 3. Front-side view of the CARE prototype and its core software architecture

Selected recommendations are forwarded to the display system. A recommendation is displayed to the user for a predefined period of time. The duration depends on the type of recommendation and its presentation format. For example, recommendations for physical exercises are typically presented for 60 seconds to give the user enough time to read the instructions and to perform the exercise. After showing a recommendation to the user, the display switches back to the picture frame mode. Currently, the system's possibilities to find out whether a recommendation has been followed are still limited. The pin board includes a set of accessories that are attached with magnetic pins. Using the magnetometer, interactions with the magnetic pins can be detected. For example, if a stretch band exercise is recommended and the magnetic field sensor detects a change in the magnetic field, the system takes this as evidence that the user took the band from the board. If the user answers to a quiz or rates a recommendation item, this information is stored in the user model. Currently, this information is not utilized in the recommendation process. However, it is used to analyse the user's preferences and the recommender system's performance and persuasiveness offline and to develop a more sophisticated recommender system.

For inter-module communication we rely on the XMPP protocol with messages in JSON format. This allows platform independent SSL encrypted communication, and facilitates integration of further modules, even if they are executed on different nodes of a computer network.

All modules produce various notifications which are centrally stored in an event log database. These log data facilitate debugging but can be exploited for evaluation purposes as well.

\section{GATHERING FEEDBACK ON CARE Us AGE}

The ultimate objective of CARE is to contribute positively to a senior person's well-being through little interventions provided during the day in the form of contextualized recommendations which are related to factors that determine well-being. The evaluation of such a system is a challenging endeavor as it raises questions such as:

- Can we reliably measure increased (or decreased) well-being as an impact of CARE usage in everyday life?

- Are the effects sustainable, or will the system's usage decrease after a certain period of time?

As a first step, we wanted to learn how a system like CARE integrates into the daily life of a senior person and whether such a system is appreciated and thus has potential impact for achieving its purpose. To this end, we did a pilot installation of our CARE prototype in a seniors' apartment, logged system usage over a period of 14 days and conducted structured interviews with the seniors after the trial.

\section{A. In-situ installation and test phase}

The in-situ evaluation of the first CARE prototype took place in the apartment of an elderly couple, a 76 years old female (named " $F$ " in the sequel), and a 75 years old male (named "M").

First, a suitable location for mounting CARE had to be found. The couple inhabits an apartment with a living room, a bed room, a bath room, a kitchen, and a small hallway. We explained to them that the system should be placed best at a location that meets the following requirements:

- CARE should be mounted in a room in which they like to dwell (e.g., the living room or the kitchen). 
- $\quad$ The CARE display should be mounted at eye level and should be easily accessible (i.e., access to CARE shouldn't be blocked by furniture or by an opened door or window).

- Access to a power socket should be available (without the need for having long extension cords).

As stated by Tolmie and Crabtree [29], developers should not decide for users where to set up an installation in order to encourage them finding out themselves how the technology could be used. Therefore we only gave the couple some hints, but left the final decision up to them where the CARE prototype should be set up. Eventually it was agreed on mounting CARE at a kitchen wall close to the passage to the living room.

The CARE staff member who launched the system advised the couple that they should not try switching off the running system or unplugging the cable from the power outlet. Instructions on how to use CARE, or comments on CARE were not given. In addition, we gave each senior a wristband equipped with an AX3 data logger, and asked them to wear the bands as often as possible since this would give us valuable data for our study. In fact, at this point we were only interested in finding out whether deployment of body-worn sensors would be a realistic option for a further CARE prototype in terms of user acceptance.

During the test period, the developers checked the system's functions twice, produced backups of recorded data and restarted the system. Except for these two interventions, the system was running continuously.

\section{B. Encounters with CARE - Usage Scenarios}

Fig. 4 and Fig. 5 give impressions on how users experienced and interacted with CARE in their apartment.

When a person is noticed in the close vicinity of CARE, the system switches from passive to active mode (cf. Fig. 4a). Such a state change is indicated to the user by swapping the displayed content - the ambient background image gets replaced by the display of a recommendation selected by the recommender engine. The recommendation remains on the screen until the user provides explicit feedback (cf. Fig. 4b), or until a timeout is reached, or in case the user is no longer in the range of CARE's sensory system for detecting the presence of a person. This is taken as that the user went away, or is at least no longer close enough to read the displayed recommendation. Note that F was wearing the AX3 wristband (cf. Fig. 4b).

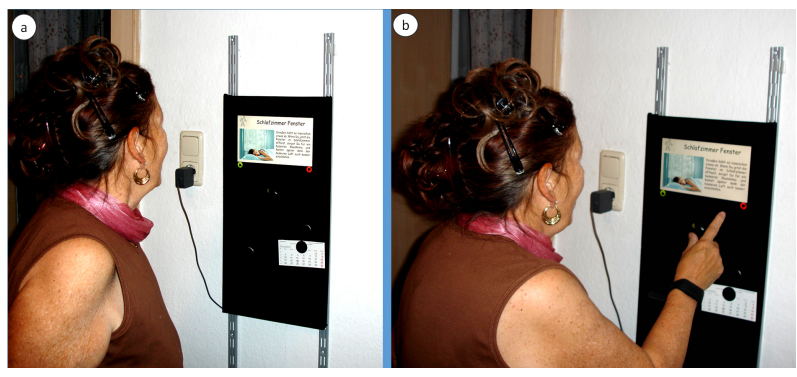

Fig. 4. Senior F reading a recommendation
Fig. 5 shows pictures taken from an encounter with CARE and the male senior $M$. After $M$ approached the installation the screen content swapped from a background image to a recommendation (cf. Fig. 5a). M showed interest (Fig. 5b) and read the recommendation, which is an advice to improve air quality, i.e., CARE recommends to air the room (Fig 5c). However, as inferable from the senior's facial expression (Fig. $5 d$ ) as well as from a negative rating of that recommendation found in the $\log$ file data., $M$ didn't appreciate much this recommendation.

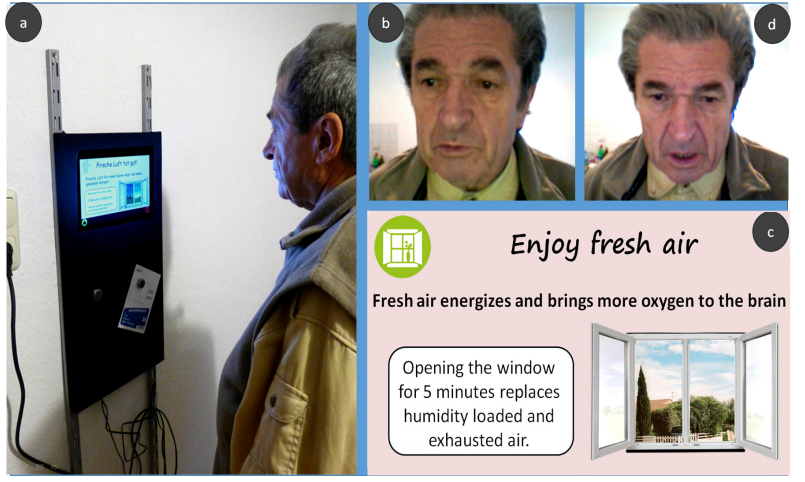

Fig. 5. Male user approaches CARE (a, b) and receives the recommendation to "air the room" (c). Apparently, the advice is negatively evaluated (d).

\section{Analysis of data logs}

During operation, the CARE system performs intensive logging of data which originate from connected sensors, and CARE system modules. All entries of CARE's internal event log database are time-stamped and listed in temporal order. In particular, the following information has been logged:

- Recommendations that have been presented,

- Assessment of a recommendation by the user,

- Self-assessment of the user's emotional state,

- Sensor readings (e.g., temperature, humidity, IR distance and 3-axis magnetometer),

- close-up images of a user's face in front of the screen - a first shot shortly after a person has approached the screen, and further ones after every 10 seconds of elapsed time of uninterrupted presence. As noted earlier, taking images was done only for the purpose of system evaluation and in consensus with the users.

The log-file data can be used to reconstruct the various user-system encounters that took place during the test period. In about $29 \%$ of the cases where user presence was detected in the IR sensor signal, the seniors dwelled in front of the display for more than two seconds so that the recommender mode was triggered. For the purpose of illustration, Fig. 6 shows readings of three sensors (IR distance, brightness, and microphone) over a selected time interval in the evening. The row labeled with "Display" indicates whether the display was in picture frame $(\mathrm{P})$ or in recommender mode (R). The interpretation of the data shown in the diagram is as follows:

At 21:59:27, a person (F) entered the kitchen and switched on the light. This can be seen as a peak in the curve of 
the brightness sensor data. A short peak in the IR distance data around 21:59:30 suggests that $\mathrm{F}$ has closely passed the screen, probably while entering the kitchen to engage in short household chores, such as putting a plate away. The noise produced by this activity can be noticed in the plot of the noise level.

The next notable raise in IR distance data at 22:00:11 indicates that $\mathrm{F}$ has approached CARE, but this time stopped in front of it. This has caused CARE to trigger the recommendation selection process. The selection of recommendations is sensitive to the current day time. Since it was already late evening, the recommender system filtered out all recommendations associated with the well-being categories physical, mental, and social. The rationale behind this filter rule is that at late evening users most probably are prepared to go to bed and thus want to come to rest, both physically and mentally. As a consequence, only recommendations of the categories environmental well-being and emotional well-being were considered for display. Recommendations for improving the room climate e.g. by airing are only given if the rating of the room climate falls below a certain threshold. This was not the case at this particular encounter. Consequently, CARE decided to recommend a relaxation exercise.

In the current prototype we rely on a simple time-out mechanism to switch back from recommender mode to picture frame mode (in case the feedback buttons are not used). As it can be seen in the "Display" row of Fig. 6, recommendations have been shown for exactly one minute, before CARE switched back to picture frame mode showing a still picture. Note that the IR distance sensor data indicate that the person dwelt for about 1.5 minutes in the close vicinity of CARE. At this point, however, we cannot directly infer from the data logs whether F was still performing the exercise or was just looking at the static image. However, as this behavior pattern occurred repeatedly, we assume that $\mathrm{F}$ was actually still engaged in the exercise even though it was no longer shown on the screen.

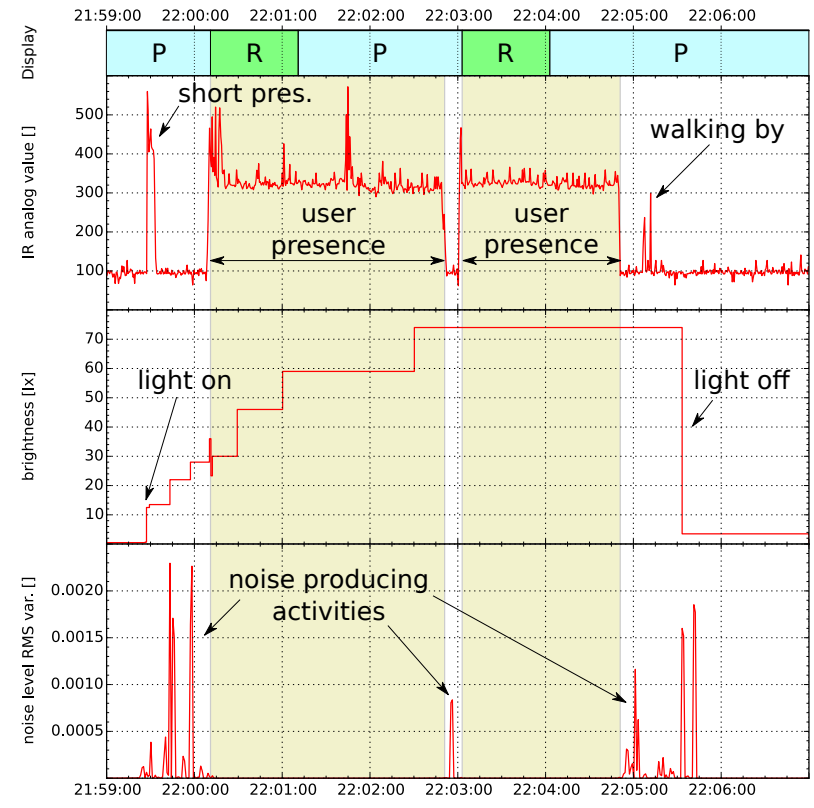

Fig. 6. Excerpt of logged sensor data
At 22:02:48, F left the detection range of the IR sensor for 12 seconds. However, increased noise level indicates that $\mathrm{F}$ was still in the vicinity of CARE. Therefore, it is less surprising, that the IR distance sensor detected her presence again at 22:03:03. Applying the filtering mechanism as explained above, CARE again recommends a relaxation exercise, but a different one this time. As shown by the logged IR distance data, $\mathrm{F}$ did dwell almost two minutes in front of CARE, despite the fact that CARE switched back to picture frame mode after 60 seconds. Again, we assume that it took F longer to finish the exercise.

At 22:04:51, F left the detection range of the IR sensor, but as suggested by the increased noise level was still active in the room. An abrupt drop of the brightness value around 22:05:40 together with a flattened noise level provide clear evidence that F switched off the light and left the kitchen.

\section{OUTCOME OF THE STRUCTURED INTERVIEW}

Immediately after the conclusion of the trial period, both seniors $(\mathrm{F}$ and $\mathrm{M})$ were interviewed separately to find out more about their experiences with the CARE display. As a basis for the interview, we started from the heuristics used by Mankoff et al. [30] for the evaluation of ambient displays and heuristics developed by Kientz et al. [31] for the evaluation of persuasive health technologies. In the following, we report on the main findings of the study using evaluation criteria from the heuristics mentioned above:

Useful and relevant information: When being asked which recommendations they could remember, the couple mentioned the physical training, the meditation exercises and the brain twisters. The couple could not remember any recommendations to improve the comfort level of their home, such as opening the window in case of bad air quality. One interpretation might be that they felt embarrassed to talk about this issue. Their preferences for specific categories of recommendations differed. $\mathrm{F}$ found the physical exercises for the fingers and hands in particular useful. She also liked the relaxation exercises where she was requested to take a deep breath or to look at the photo of a wide countryside and have the colors take an effect on her. She mentioned that she knew that some people were doing relaxation exercises, but never thought that this is something she could do herself. However, she also said that she would like to conduct longer exercises on a regular basis, about 20 to 30 minutes, twice a day. Overall, the duration of the interaction was considered too short. $\mathrm{M}$ felt most attracted by the jokes. Both $\mathrm{F}$ and $\mathrm{M}$ emphasized that a larger variety of content should be displayed.

Appropriate Time and Place: F and M agreed that the kitchen was an appropriate place to install CARE. Since they had to go in and out the kitchen many times a day, information was provided at an opportune time and place. However, $\mathrm{F}$ also said that she would prefer longer interactions with the system twice a day (in the morning and in the evening) to conduct one exercise after the other as opposed to many interactions during the day. $\mathrm{M}$ did not mention any timing issues. A reason might be that he was less interested in the physical exercises for which most of the timing issues were observed.

Ease of Use: $F$ and $M$ agreed that the images and the text were well readable. Furthermore, they found the amount 
of information per screen appropriate. They also said that the meaning of the buttons that could be selected was clear to them. Even though user intervention was kept at a minimum, we observed a number of usability issues. Due to some technical issues with the person detector, the CARE system did not always react promptly to an approaching person. As a result, $\mathrm{F}$ and $\mathrm{M}$ sometimes moved away from the display before the information was displayed. Simple feedback should be given when a person has been detected. In some cases, it happened that the user slightly moved away from the display without intending the presentation to be stopped.

Flexibility and Efficiency of Use: In order not to overburden the users, we tried to keep the interface as simple as possible. In particular, we did not require the users to navigate back and forth on the display. However, both $\mathrm{F}$ and $\mathrm{M}$ were asking for more flexibility to control the display. F mentioned that some exercises required a specific appliance, such as a ball or a chair, which she had to get first. Also she got sometimes interrupted by her partner asking her for something during an exercise. In such situations, she would like to suspend a physical exercise and resume at a later time.

Peripherality of display: Even though the display was permanently running, neither $\mathrm{F}$ nor $\mathrm{M}$ found the display disturbing. This is in contrast to studies by Consolvo and Towle [32] who observed in an in-situ study that their ambient display was considered distractive in a dark room because it was glowing brightly. In our case, the problem did not arise because the display was installed in the kitchen which was not used in low-light conditions unlike a TV or bed room.

Educating users: Users should understand why the actions they do contribute to their well-being. F had a clear understanding of the purpose of the presented exercises. Her knowledge stems from her participation in a rehabilitation program and her previous work as a care taker in a home for elderly people.

Appropriate Functionality: Both $\mathrm{F}$ and $\mathrm{M}$ stated that the CARE system integrated well in their daily routine. In particular, F was positively impressed by the CARE system. She said that CARE helped her stay active and prevented her from getting lazy. As a reason, we indicate that $F$ had a higher intrinsic motivation than $\mathrm{M}$ to improve her level of fitness. Furthermore, the displayed content, in particular the physical training and relaxation exercises, matched her personal needs in a better manner. There was also evidence that $\mathrm{F}$ had a clearer understanding of the purpose of the presented recommendations than $\mathrm{M}$.

\section{LESSONS LEARNT AND OUTLOOK}

From analyzing log file data of the in-situ trial and the conducted post-test interview we got a number of valuable insights and inspirations for our further work. The episodes presented in Section IV-C showed how sensor data can be exploited for analyzing CARE usage and also for identifying characteristic usage patterns. In addition, we got a better understanding of the limitations of CARE's current sensor system which will help us fine-tune parameter settings, e.g., for noise cancellation in sensor data. From our observations we compiled a list of requirements which will guide our work towards an extended CARE prototype.
Give users more control: In order to ensure that the CARE system was easy to use by elderly people, we focused on information that can be sensed without requiring a large amount of user interaction. It turned out, however, that the users wanted more control over the interface. In particular, better mechanisms are required to adapt the timing of the presentations to the users' needs and preferences. Therefore, we plan to investigate how to give users more options to customize the interface.

Improve usability of touch-based interface: To adapt the CARE system continuously to the users' preferences and needs and to evaluate the effect of the CARE system on the users' well-being, we tried to encourage users to give feedback on the quality of recommendations and their current mood by pressing specific buttons. Even though our users said that they understood the meaning of the buttons, they did not often use the opportunity to provide feedback. By analyzing accelerometer sensor data, we noticed that the display had been touched by the elderly without the system capturing the users' input. Probably, the users encountered similar timing issues during tapping as reported in [17].

Include incentives for users: So far, we did not include a large number of incentives to increase the chances that recommendations are followed. While the purpose of the recommendations was clear to the users, they need to be linked more explicitly to their overall goals. In particular, users should be able to monitor their progress on set goals. Other options to increase motivation we discussed with the seniors included the integration of concepts from gamification and social media.

Enhance the sensibility of CARE: To improve the quality of the selected recommendations, the potential of mechanisms for context and activity recognition needs to be exploited more effectively considering both stationary as well as wearable sensors. In particular, CARE should be able to learn which kinds of recommendations are successful in which context. A more sophisticated version of CARE may consider additional factors for the selection of recommendations, such as the nature of acquired context information, the user model, and the discourse history that keeps a record of what has been shown to the user and whether user feedback was received. If CARE is used by more than one user, robust methods for automated user identification will be required for personalization. Currently, CARE relies on a repertoire of manually pre-authored recommendations typically consisting of the actual recommendation and a motivating picture and/or text. However, to allow for a greater amount of adaptation, recommendations should be automatically composed depending on the individual user and the current context.

Increase the repertoire of recommendations: In order to keep users engaged over a longer period of time, the repertoire of recommendations needs to be enhanced. Options to explore include the use of online resources (such as sites providing tips for healthy cooking) in order to provide users with more serendipitous encounters. In addition, taking account of the user's social well-being, we plan to allow family members to provide input, e.g., by posting images and greetings via a web interface or social media in order to enhance feelings of connectedness and promote reciprocity [23]. 
Paper accepted for 2015 International Conference on Pervasive Computing Technologies for Healthcare (PervasiveHealth). Personal use of this material is permitted. Permission from IEEE must be obtained for all other uses, in any current or future media, including reprinting/republishing this material for advertising or promotional purposes, creating new collective works, for resale or redistribution to servers or lists, or reuse of any copyrighted component of this work in other works.

\section{ACKNOWLEDGMENT}

This work is supported by the German Federal Ministry of Education and Research (BMBF) under grant number $01 \mathrm{XZ13022}$ and carried out jointly with a sister project at the ICCS of the National Technical University of Athens.

\section{REFERENCES}

[1] F. Sun, I. J. Norman, and A. E. While, "Interventions targeting social isolation in older people: A systematic review," BMC Public Health, vol. 13, 2013.

[2] T. Schmid, Ed., Promoting Health Through Creativity: For Professionals in Health, Arts and Education. Whurr Publishers, 2005.

[3] B. Arnetz and T. Theorell, "Psychological, sociological and health behaviour aspects of a long term activation programme for institutionalized elderly people," Social Science and Medicine, vol. 17, pp. 449-456, 1983.

[4] M. Cattan, M. White, J. Bond, and A. Learmouth, "Preventing social isolation and loneliness among older people: A systematic review of health promotion interventions," Ageing \& Society, vol. 25, pp. 41-67, 2005.

[5] N. D. Lane, M. Lin, M. Mohammod, X. Yang, H. Lu, G. Cardone, S. Ali, A. Doryab, E. Berke, A. T. Campbell, and T. Choudhury, "Bewell: Sensing sleep, physical activities and social interactions to promote wellbeing," Mob. Netw. Appl., vol. 19, no. 3, pp. 345-359, 2014

[6] S. Consolvo, D. W. McDonald, T. Toscos, M. Y. Chen, J. Froehlich, B. Harrison, P. Klasnja, A. LaMarca, L. LeGrand, R. Libby, I. Smith, and J. A. Landay, "Activity sensing in the wild: A field trial of ubifit garden," in Proc. of the SIGCHI Conference on Human Factors in Comput. Systems. New York, NY, USA: ACM, 2008, pp. 1797-1806.

[7] F. Bentley, K. Tollmar, P. Stephenson, L. Levy, B. Jones, S. Robertson, E. Price, R. Catrambone, and J. Wilson, "Health mashups: Presenting statistical patterns between wellbeing data and context in natural language to promote behavior change," ACM Trans. Comput.-Hum. Interact., vol. 20, no. 5, pp. 30:1-30:27, Nov. 2013.

[8] Y. Lin, J. Jessurun, B. de Vries, and H. Timmermans, "Motivate: Context aware mobile application for activity recommendation," in Proc. of the Second International Conference on Ambient Intelligence. Berlin, Heidelberg: Springer-Verlag, 2011, pp. 210-214.

[9] M. Turunen, J. Hakulinen, C. Smith, D. Charlton, L. Zhang, and M. Cavazza, "Physically embodied conversational agents as health and fitness companions," in INTERSPEECH 2008, 9th Annual Conference of the International Speech Communication Association, Brisbane, Australia, September 22-26, 2008. ISCA, 2008, pp. 2466-2469.

[10] U. Yasavur, C. Lisetti, and N. Rishe, "Lets talk! speaking virtual counselor offers you a brief intervention," Journal on Multimodal User Interfaces, vol. 8, no. 4, pp. 381-398, 2014.

[11] L. Ring, L. Shi, K. Totzke, and T. Bickmore, "Social support agents for older adults: Longitudinal affective computing in the home," Journal on Multimodal User Interfaces, vol. 9, no. 1, pp. 79-88, 2015.

[12] L. P. Vardoulakis, L. Ring, B. Barry, C. L. Sidner, and T. Bickmore, "Designing relational agents as long term social companions for older adults," in Proc. of the 12th International Conference on Intelligent Virtual Agents. Berlin, Heidelberg: Springer, 2012, pp. 289-302.

[13] L. Angelini, M. Caon, S. Carrino, L. Bergeron, N. Nyffeler, M. JeanMairet, and E. Mugellini, "Designing a desirable smart bracelet for older adults," in Proc. of the 2013 ACM Conference on Pervasive and Ubiquitous Computing Adjunct Publication. New York, NY, USA ACM, 2013, pp. 425-434.

[14] R. Leung, C. Tang, S. Haddad, J. Mcgrenere, P. Graf, and V. Ingriany, "How older adults learn to use mobile devices: Survey and field investigations," ACM Trans. Access. Comput., vol. 4, no. 3, pp. 11:111:33, Dec. 2012.

[15] T. Jones, D. Kay, P. Upton, and D. Upton, "An evaluation of older adults use of ipads in eleven uk care-homes," Int. J. Mob. Hum. Comput. Interact., vol. 5, no. 3, pp. 62-76, Jul. 2013.
[16] L. G. Motti, N. Vigouroux, and P. Gorce, "Interaction techniques for older adults using touchscreen devices: A literature review," in Proc. of 25ème Conférence Francophone sur L'Interaction Homme-Machine. New York, NY, USA: ACM, 2013, pp. 125:125-125:134.

[17] C. Leonardi, A. Albertini, F. Pianesi, and M. Zancanaro, "An exploratory study of a touch-based gestural interface for elderly," in Proceedings of the 6th Nordic Conference on Human-Computer Interaction: Extending Boundaries, ser. NordiCHI '10. New York, NY, USA: ACM, 2010, pp. 845-850.

[18] V. Mateevitsi, K. Reda, J. Leigh, and A. Johnson, "The health bar: A persuasive ambient display to improve the office worker's well being," in Proc. of the 5th Augmented Human International Conference. New York, NY, USA: ACM, 2014, pp. 21:1-21:2.

[19] C. Obermair, W. Reitberger, A. Meschtscherjakov, M. Lankes, and M. Tscheligi, "perframes: Persuasive picture frames for proper posture," in Proc. of the 3rd International Conference on Persuasive Technology. Berlin, Heidelberg: Springer, 2008, pp. 128-139.

[20] J. Rowan and E. D. Mynatt, "Digital family portrait field trial: Support for aging in place," in Proceedings of the SIGCHI Conference on Human Factors in Computing Systems, ser. CHI '05. New York, NY, USA: ACM, 2005, pp. 521-530.

[21] S. Consolvo, P. Roessler, and B. E. Shelton, "The carenet display: Lessons learned from an in home evaluation of an ambient display," in UbiComp 2004: Ubiquitous Computing: 6th International Conference, Nottingham, UK, September 7-10, 2004. Proceedings, ser. Lecture Notes in Computer Science, N. Davies, E. D. Mynatt, and I. Siio, Eds., vol. 3205. Springer, 2004, pp. 1-17.

[22] S. E. Lindley, R. Harper, and A. Sellen, "Desiring to be in touch in a changing communications landscape: Attitudes of older adults," in Proceedings of the SIGCHI Conference on Human Factors in Computing Systems, ser. CHI '09. New York, NY, USA: ACM, 2009, pp. 1693-1702. [Online]. Available: http://doi.acm.org/10.1145/1518701.1518962

[23] R. Cornejo, M. Tentori, and J. Favela, "Enriching in-person encounters through social media: A study on family connectedness for the elderly," Int. J. Hum.-Comput. Stud., vol. 71, no. 9, pp. 889-899, 2013. [Online]. Available: http://dx.doi.org/10.1016/j.ijhcs.2013.04.001

[24] M. López-Nores, Y. Blanco-Fernändez, J. J. Pazos-Arias, and J. GarcíaDuque, "Exploring synergies between digital tv recommender systems and electronic health records," in Proceedings of EuroITV '10. New York, NY, USA: ACM, 2010, pp. 127-136.

[25] T. Taneda, M. Lee, and K. Pollard, "Scl/prb index of wellbeing in older populations," Stanford Center on Longevity, Tech. Rep., Juni 2001. [Online]. Available: http://www.prb.org/pdf11/SCLPRBIndexofWellBeing.pdf

[26] C. Fan, J. Forlizzi, and A. Dey, "Considerations for technology that support physical activity by older adults," in Proc. of the 14th International ACM SIGACCESS Conference on Computers and Accessibility. New York, NY, USA: ACM, 2012, pp. 33-40.

[27] A. Seiderer, S. Hammer, E. André, T. Rist, and M. Mayr, "Exploring digital image frames for lifestyle intervention to improve well-being of older adults," in Proceedings of the 5th International Conference on Digital Health. New York, NY, USA: ACM, 2015, pp. 639-648.

[28] G. Adomavicius and A. Tuzhilin, "Context-aware recommender systems," in Recommender Systems Handbook, F. Ricci, L. Rokach, B. Shapira, and P. B. Kantor, Eds. Springer US, 2011, pp. 217-253.

[29] P. Tolmie and A. Crabtree, "Deploying research technology in the home," in Proceedings of the 2008 ACM Conference on Computer Supported Cooperative Work, ser. CSCW '08. New York, NY, USA: ACM, 2008, pp. 639-648.

[30] J. Mankoff, A. K. Dey, G. Hsieh, J. Kientz, S. Lederer, and M. Ames, "Heuristic evaluation of ambient displays," in Proc. of CHI '03. New York, NY, USA: ACM, 2003, pp. 169-176.

[31] J. A. Kientz, E. K. Choe, B. Birch, R. Maharaj, A. Fonville, C. Glasson, and J. Mundt, "Heuristic evaluation of persuasive health technologies," in Proc. of the 1st ACM International Health Informatics Symposium, ser. IHI '10. New York, NY, USA: ACM, 2010, pp. 555-564.

[32] S. Consolvo and J. Towle, "Evaluating an ambient display for the home," in CHI '05 Extended Abstracts on Human Factors in Comput. Systems. New York, NY, USA: ACM, 2005, pp. 1304-1307. 